\title{
Adsorption de protéines à une interface eau/air fonctionnalisée
}

\author{
Proteins adsorption at a functionnalised air/water interface
}

\author{
Nicolas Blondiaux*, I,aurent Davoust ${ }^{1 *}$, Jean Berthier**, Dominique Masse**, Frédéric (Ginot*** \\ *LEGI. Equipe PIM. BP 53, 38041 Grenoble cedex 9 \\ *:*ETT, CEA-Technologie Avancées, BP 53, Grenoble cedex 9 \\ ***BIO-MERIEUX. CEA-Technologie Avancées, BP 53, Grenoble cedex 9
}

\begin{abstract}
An optical set-up coupled to a microscope is developed in order 20 follow the aging kinetics of a planar air/water interface from induced fluorescence. The technique is characterized with fluorescent-lagged particles and firther applied to an airhwater interface functionalized to adsorb selectively strepavidin. The second configuration under study turms out to be a 2-D foam entrapped between no glass plases; a modelling saking inso account diffusion and adsorption is solved by finite element method.
\end{abstract}

\section{I INTRODUCTION}

Les systìmes de détection de molécules biokgiques actucis (hio-puces) lont intersenir un support solide (en verre ou silicium) préaliablement lonctionnalisé à l'aide de sites moléculaires récepteurs. 1 "échantillon liquide à analyser est mis en contact avec le support fonctionnalisé : si les molécules cibles à capplurer sont en quantité suffisante dans léchantillon liquide, un certain nombre dentre elles $s$ advorbe at la surliace par diffusion puis reconnaissance spécifique avec les sites récepleurs. Pour la grande majorité des hio-puces. la distribution des récepteurs est discrète et la surlace fonctionnalisée présente une extension tinie: ce qui conduil à une sensibilité de délection limitéc.

Cel arlicle s'inlegre dans une strattégie |11 yui consiste is fonctionnaliser non plus une interface solide/liquide mais unc interlace liquide/gaz pour i) aklsorber/concentrer des molécules cibles. ii) labricuer de l'aire interfaciale en produisant une mousse. Less caracléristiques de chasque configuration (interlace plane ou mousse) sont rappelées dans le lableau suivant: les moyens de modélisattion ou de délecelion associés font intervenir des phénomènes multi-physiques caracléristiques de la microfluidique: importance de laadsorption moléculaire, rhéologic interliaciale. détection par le potentiel électrique de surfice... les points ahordés dans eel article sont soulignés dans le tableatu : ils se rassemblent atulour d'un objectif conmun : développer une méthode optique générique permettant destimer l'adsorption de molécules cibles a une interface et dans une mousse 2-D constiluées toutes deux d'ealu el d'air. L'interface eau/air constille un sile privilégić pour l'adsorption d'objets amphiphiles (détergents. lipides. colloïdes).

\begin{tabular}{|c|c|c|c|}
\hline & Détection des cibles & Modélisation & Avantages $(+) /$ Inconvénients (-) \\
\hline $\begin{array}{l}\text { Interface } \\
\text { plane }\end{array}$ & $\begin{array}{l}\text { - Electrique (fortentiel de surfiace) } \\
\text { - Optique (BAM. ellipsométrie, microscopie } \\
\text { optique, fluorescence) } \\
\text { - Rhéologique (tension de surfitce) }\end{array}$ & $\begin{array}{l}\text { I-D : voir lintéralure } \\
|2.3|\end{array}$ & $\begin{array}{l}\text { (+) Géométrie el modélisation simples } \\
(-) \text { Surliace fonctionnaliséc finic }\end{array}$ \\
\hline .nousse 2-D & $\begin{array}{l}\text { - Rhéologique (élasticité) } \\
\text { - Optique (microscopie optique, } \\
\text { fluorescence) }\end{array}$ & $\begin{array}{l}\text { 2-D : modélisătion } \\
\text { spécifíque }\end{array}$ & $\begin{array}{l}\text { (+) Surlace fonclionnaliséc } \\
\text { (+) Etficacité d’adsorption } \\
\text { (-) Modélisidion délicate } \\
\text { (-) Géométric complexe }\end{array}$ \\
\hline
\end{tabular}

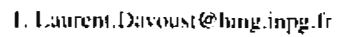


Des expériences sont présentées avec l'exemple de particules fluorescentes et d'une protéine très répandue en biologie : la streptavidine. Les précisions sur la fonctionnalisation physicochimique de l'interface pour l'adsorption de cette protéine sont apportées. Finalement. une modélisation des phénomènes de diffusion/adsorption de molécules cibles est proposée pour une mousse 2-D eau/air.

\section{II $\square$ ADSORPTION À UNE INTERFACE EAU/AIR PLANE}

Pour valider la fonctionnalisation d'une interface eau/air, il faut mesurer son efficacité d'adsorption pour les cibles choisies. La stratégie retenue est une technique de fluorescence induite qui se décompose en trois ćtapes : l'excitation

Filtre pour

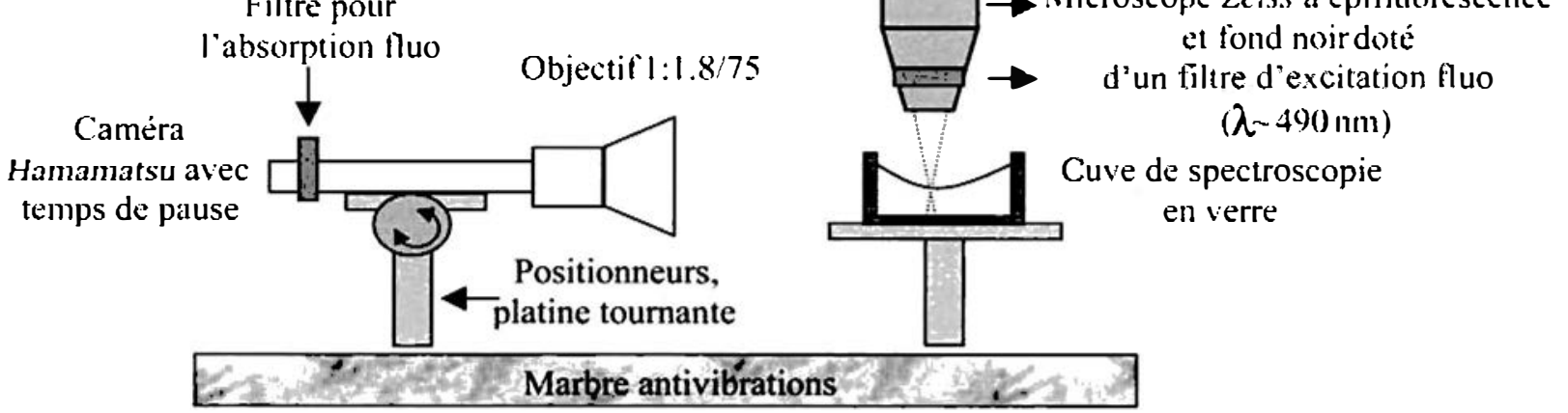

Figure 1 : Montage optique pour les mesures de vieillissement de l'interface eau/monocouche/air fonctionnalisée.

Le but est de mesurer l'augmentation de signal fluorescent provenant de l'interface pendant l'adsorption de molécules cibles fluorescentes tout en surveillant la surface fonctionnalisée (évolution de structurations...). Une cuve de spectroscopic contient un échantillon liquide à la surface duquel est élalée une monocouche de phospholipides (fluorescents). L'émission fluorescente ou l'éclairage en lumière blanche tangentiel (fond noir) sont assurés par un microscope Zeiss. La première solution pour visualiser la fluorescence de l'interface est d'utiliser l'objectif du microscope mais la lluorescence de molécules solubilisćes en sous-phase vient se superposer à la contribution de l'interface. Pour s'affranchir de cette difficulté, un dispositif de visualisation latérale est installé. Le microscope réalise donc un faisceau de diamètre et de divergence réglables grâce à ses différents diaphragmes et objectifs. Un filtre permettant de régler la longueur d'onde d'excitation fluo est inséré juste avant l'objectif du microscope. Le faisceau obtenu est plus ou moins conique (selon l'objectif sélectionné) et son diamètre reste faible par rapport aux dimensions de la cuve (réglage par le diaphragme et l'objectif sélectionnés). Le diaphragme permet également de régler l'homogénéité en intensité lumineuse du faisceau émis. Le dispositif de capture latérale comprend une caméra, un objectif, des bagues allonges et un filtre dont le spectre est adapté au fluorophore. L'ensemble est monté sur un positionneur 3 axes et possède un réglage d'inclinaison.

l.es filtres sont caractérisés au spectrophotomètre afin de vérifier si leurs spectres ne s'entrecoupent pas et s’ils sont du fluorophore. le temps de vie de l'état excité et la désexcitation du fluorophore (émission d'un photon) |4|. Chaque fluorophore possède un spectre d'absorption et d'émission. Il est préférable d'exciter le fluorophore avec une longueur d'onde correspondant à son pic d'absorption tout en prenant garde :

- au photobleaching : si le fluorophore est illuminé trop intensément, il est tout simplement détruit.

- au quenching : lorsque le fluorophore émet un photon par fluorescence, celui-ci peut aller exciter une molécule voisine plutôt que de partir vers le photodétecteur.

- à toute émission de fluorescence parasite provenant de matćriaux comme les plastiques.

II.1 Description du montage (figure I) 


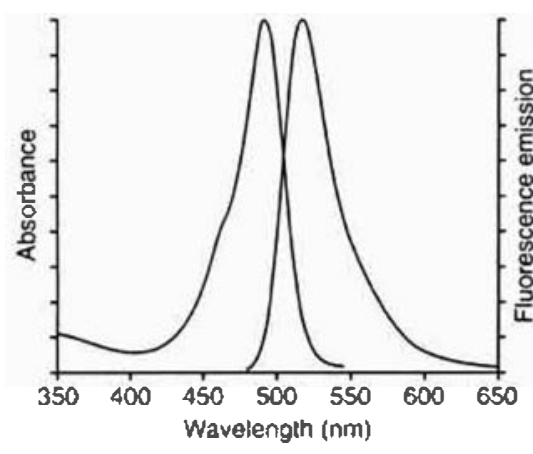

a)

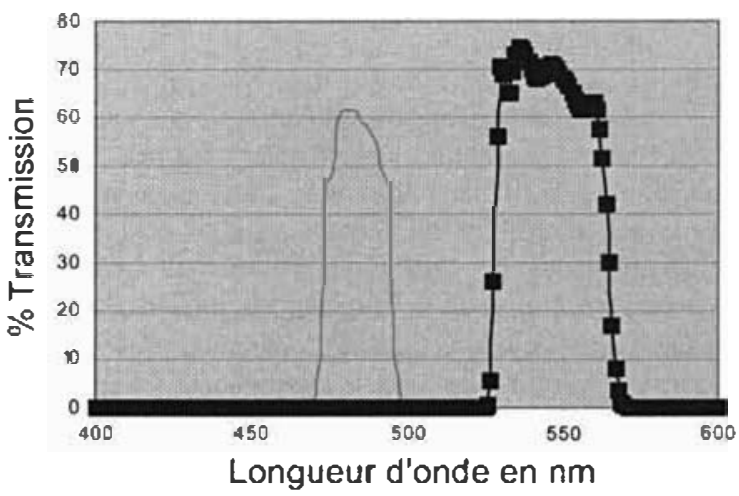

b)

Figure 2 : a) Spectres d'adsorption et d'émission de fluorescence des particules Transfluo. b). Spectres d'excitation (-) et d'absorption (-) des filtres impliqués dans le montage optique.

fréọuence spatiale donnće. Puisque la CCD de la caméra mesure $1 \mathrm{~cm}$ de large. on en déduit un grossissement de 3 . La profondeur de champ esi la distance séparant deux points extrêmes de l'axe optique du dispositif dont les images sont vues avec une netceté suffisisante : elle dépend du grossissement du dispositif. de son ouverture numérique et de la taille des pixels du capteur. Plus délicate à détenniner, elle laait intervenir la notion de flou : dans notre application, elle peut être estimée à 300$) \pm 50 \mathrm{~mm}$. Cette grandcur doil ensuite être comparée à la taille du faisceau: si clle est plus grande que le diamèlre du faisceau (objectit de lor grossissement), lout ce qui est éclairé apparaitra net sur li caméra. Dans le cas contraire (faisceau d’éclairage de grand diamètre), seule une partie de l'image sera nette (situalion incésirable).

\section{- Localisation de la surface}

L.image obtenuc par lc dispositif de capture latérale est assez complexe car le liquide de la cuve forme un ménisque. La cuve élanı carrée el le verre utilisé hydrophile, on obtient un ménisque de forme concave avec une zone approximativement plane au centre de la cuve. La première expérience réalisće pour localiser l'interface est réalisće avec des phospholipides fluorescents (NBD-PE, fournisseur: Avanti Polar Lipid). De par leur caractère amphiphile, ils se trouvent naturellement à l"interface et en délivrent ainsi une image précisc sur la caméra CCD. La figure 3 montre comment. selon la position du faisceau incident par rapport au centre de la cuve, une ellipse plus ou moins aplatie se dessine qui figure l' interface. En fait. l'élémenı surfacique éclairé par le faisceau peut être plus ou moins incurvé selon sil position parr rapport à l'interlace considérée. Dans la région centrale de l'interface (la plus planc), l'ćtendue géométrique selon l'axe $\mathrm{z}$ de l'élément surfacique éclairé est quasiment nulle. En revanche, si le faisceilu s'approche du bord de la cuve, la courbure de l'interface augmente et en première approximation. on peut considérer que l'élément surfacique éclairé réilise l'intersection d'un cylindre avec une surface concave.

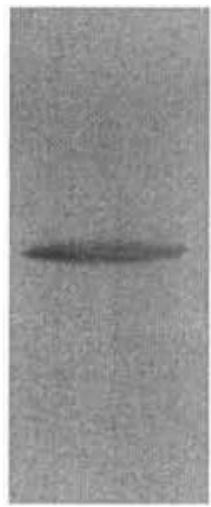

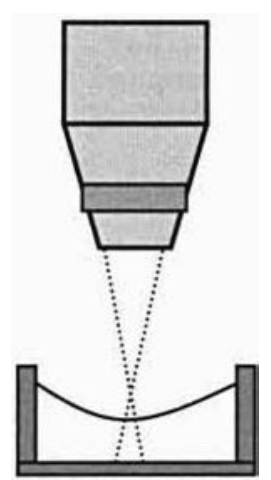

a)
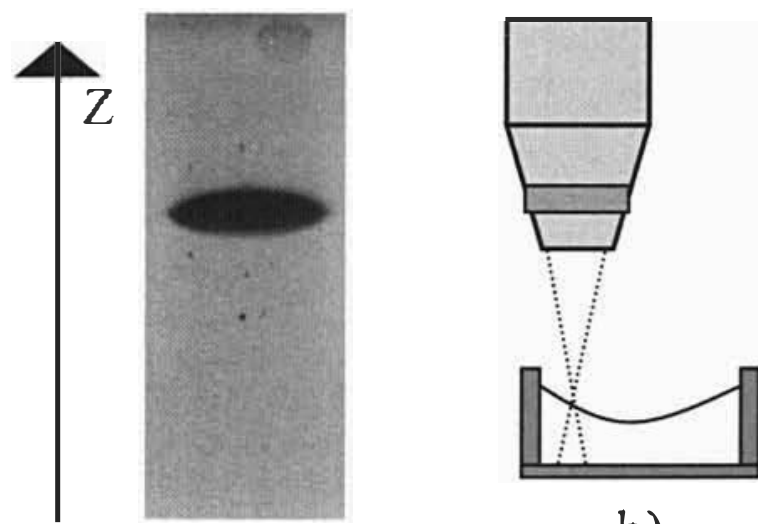

b)

Figure 3 : Localisation de l'interface par des phospholipides fluorescents : a) éclairage au centre de l'interface, b) éclairage à la périphéric de l'interface. 


\section{II.3 Cinétique d'adsorption pour des particules fuorescentes}

La deuxième expérience de validation du montağe est réalisée à l'aide de particules Trunsfluo ( $): 0.1 \mu \mathrm{m}$, Molecular Probes). Ces particules présentent certains avantages par rappor1 aux molécules lluoreșcentes: elles sont hydrophobes. ce qui permet de s'affranchir de la fonctionnalisation de l'interface eau/air. et se distinguent les unes des autres sur l'image. Une solution aqueuse contenant des particules Transfluo est introduite dans la cuve et une image est produite sur la caméra latérale. Cette fois. la sous-phase est également thuorescente (figure 4). La zone inférieure correspond aux rayons émis par les particules de la sous-phase (voir cas $n^{\circ} \mid$ ci-après). L'cllipse très lumineuse provient des particules à l"inlerface. La zone lumineuse supérieure est un autefact correspondant à l'inage de la sous-phase à travers le dioptre que constitue l'interface. Quelques considérations d’optique géométrique sont nécessaires pour voir l’origine de cette demière interprétation (figure 5 ).

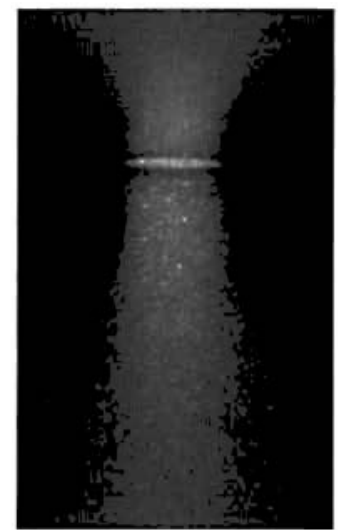

Figure 4 : Image obtenue pendant l'adsorption de particules Transfluo.

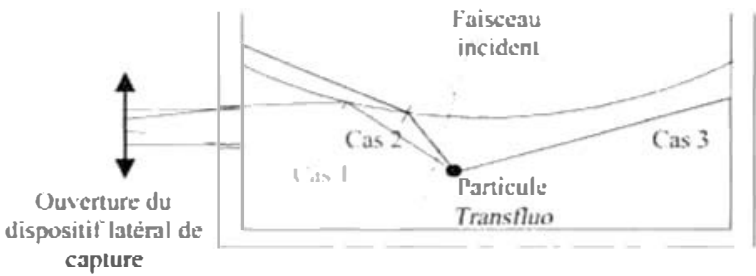

Figure 5 : Trois trajectoires possibles pour des rayons lumineux émis à partir d'un fluorophore en sous-phase.

- cos $n^{\circ} /$ : les rayons émis par le tluorophore à partir de lá sous-phase traversent le liquide. sortent de la cuve puis sont captés par la caméra CCD.

- cas $n^{\circ} 2$ : les rayons sont émis vers l'interlace. Leurs angles d'incidence sur l'interface sunt tels qu il y a réllexion totale (indice de l'eau supéricur à celui de l'air, angle de réflexion totale de $41,8^{\circ}$ ). Ces rayons ainsi déviés sont ensuite captés par la CCD.

- $\cos n^{\circ} 3$ : les rayons sont perdus.

Le cas 2 entraîne donc la formation d'une seconde image sur la CCD. Au final. cet artefact šavère très utile pour s'assurer du bon emplacement de l'interlace.

\section{- Traitement d'image et validation du montage}

Pour valider le montage optique proposé, une cinétique d'adsorption est finalement réaliséc avec les particules Transfluo. Un traitement d'image est réalisé afin d'isoler le signal spécifique de l'interlace: le niveau de gris moyen intégré sur l'ellipse. Le diagramme ci-après présente les dilférentes élapes du traitement d’image réalisé sur Mallab.

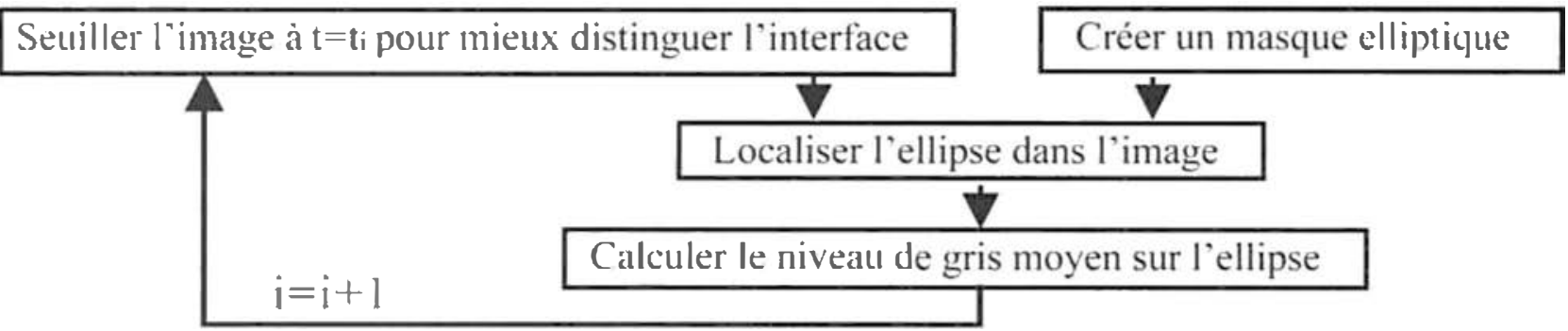

Le signal de fluorescence provenant de l'interface augmente notablement comme le montre les clichés de la figure 6. Une fois le traitement d'image réalisé, nous obtenons pour ces particules la courbe de cinétique présentée sur la figure 7 qui montre bien l'augmentation du signal d'adsorption au niveau de l'interface ainsi qu'un processus de saturation explicable par le fait que la concentration interfaciale en particules Transfluo a atteint si valeur maximale $\left(\Gamma \sim \Gamma_{\infty}\right.$ en $\left.\mathrm{mol} / \mathrm{m}^{2}\right)$.
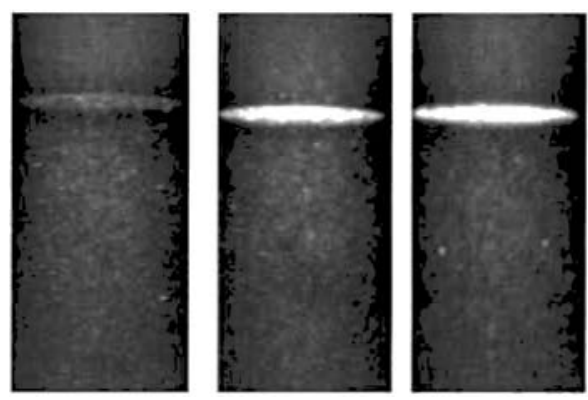

Figure 6 : Images obtenues pendant le processus d'adsorption des particules Transfluo à 3 instants différents : $\mathrm{t}=20 \mathrm{mn}, 40 \mathrm{mn}, 60 \mathrm{mn}$. 


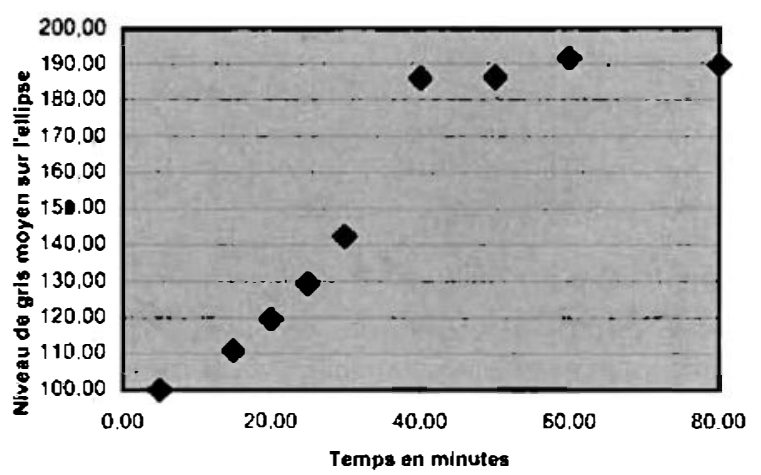

Figure 7 : Courbe de cinétique ubtenue après traitement d'inage.

\section{III - INTERF $\Lambda$ CE EAU/AIR FONCTIONNAIJISEE.}

\section{s) III.1 Montage moléculaire}

Si l'on souhatile capturer une mulécule cible non spécialement illuphiphile. il est nécessaite de créer une aflinité chimicue entre cetle-ci el l'intertice call/sir support. Cetle derniere est donse lenctionnalisce à l'aiche d'une couche de molécules amphiphiles ayant des sites chimiques spécilicyues de lia cible a capturer par adsurption.

Lit molécule cible choisic dans cette cutude est la streptavidine $|5|$ : cest une proléine. soluble dans l'satu. bien connue en hislugie moléculaire. Les molécules itmp̨hiphiles choisies dins celle élude sont des phospholipides insolubles datns lesau qui pusiedent une léte polaire hydrophile reliec à deux chaînes carbunées hydrophobes. I.orsqu'ils som étalés sur l'eitls. ils printent leur lête hydruphile vers l'eilu ssousphisel el laissent leurs queues hydrophobes hors de l'ealu dans l'air (figure $\delta$ ) : ils sorganisent sous la forne d'un fïlm plus ou moins lluicle selon leur concentration surfacique (phase gitzuse. licpuide on solide, figure 9). Pour achever la fonctionnalisation de la surlitede, lat strattégie adoptéc consiste ì gretler au niveiau de la téle hydruphile de ces phospholipides une molécule de hiotine. Lin lel montage moléculaire fait l'objel d'un trisail d’ingenierie spécitique : ces phospholipides "biotinylés" sont done directement ichetés (BiotineDiPalmitoil-PhosphattidyleEshanolamine ou BDPPE. fournisseur : Aremi Poler lipief). Les phospholipides biotinylés presents à l'inlertike edu/air fonctionnalisent celle-ci pour lit streptavidine via la réation streptavidine-hiotine. la biotine tou vitimine $H$ ). remplit donc un role de médiateur: elle possede l'arlinité recherchée pour lés siles récepteurs de lat streptavidine. Lil réatcion de reconnaissance moléculaire entre streptavidine et biotine. de type elé-serrure est tres

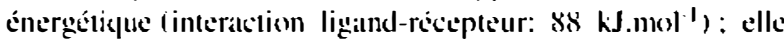
est caractériséc par une constante d'aflinité très élevée $K_{i l}=1()^{+15} \mathrm{M}^{\prime}$ qui traduil le fait que l'alssociation hiotinestreplavidine n'est que très peu réversible. Finalement. les phospholipides hootinylés sont mélangés aveé des phospholipides neutres et intercalants (DiOlenyl-PhosphatidileCholine uu DOPC, lournisseur: Sigme-A/drich) qui permettent de conserver la diffusion interliciak dans la monocouche si des molécules de streptavidine sont adsorbées vigure \&).

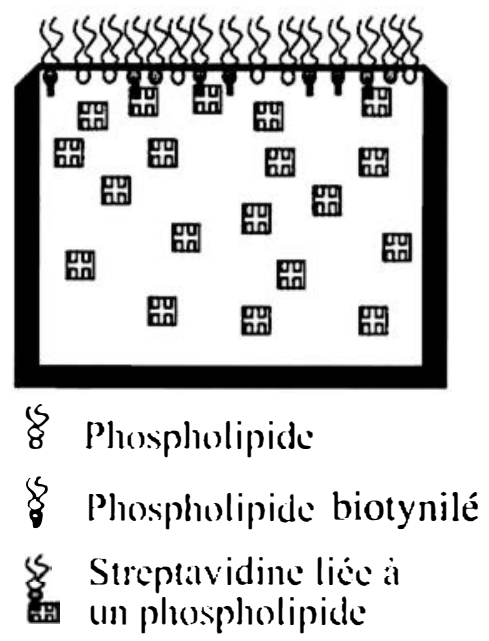

Figure 8 : Interface cau/air finnctionnaliscée.

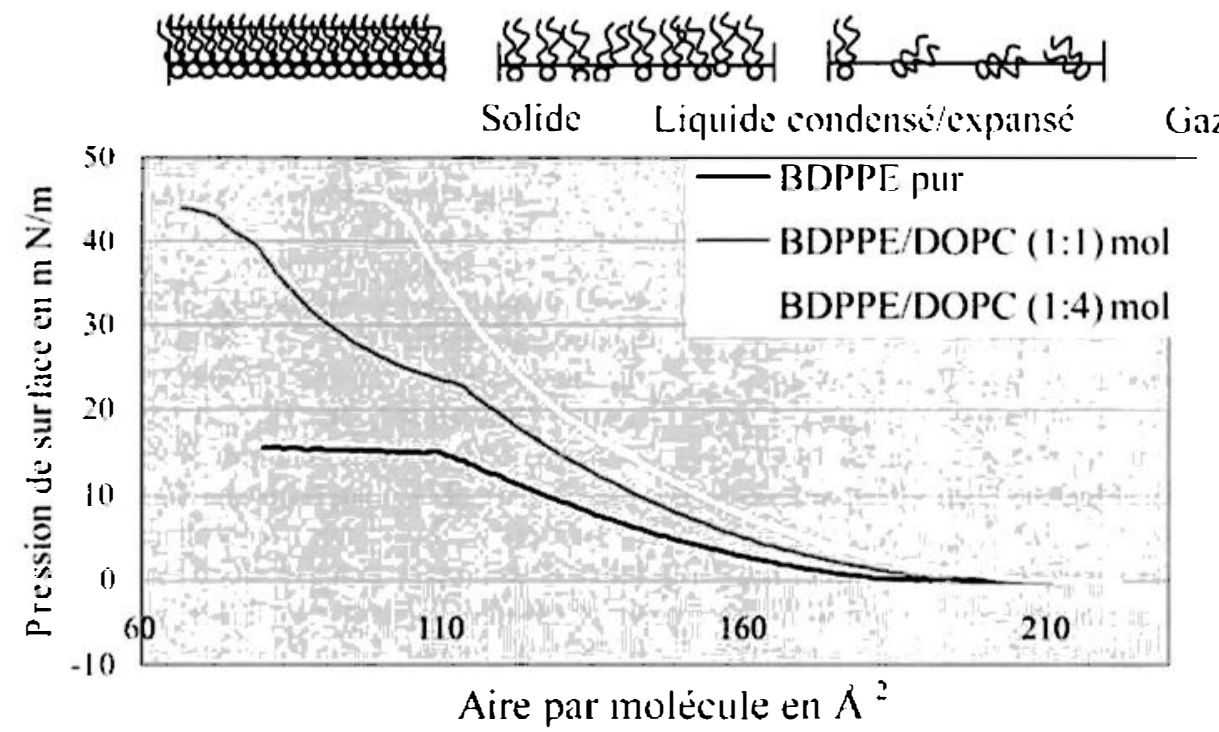

Figure 9 : Isothermes de compression réalisées pour une monocouche de phosphoulipides biotinylés (BDPPE), une monocouche mixte constituéc de BDPPE et phospholipides intercalants (DOPC) avec deux rapports de dilution. 


\section{III.2 Fabrication ct caractérisation d'un film de phospholipides}

Le tilm est fabricué par dénòt(s) sur la sous-phase ayueuse de quelques $\mathrm{ml}$ d'un solvant organique volatile (chloroforme. hexine) contenatnl les phospholipides dilućs. La natture du solvant et ta concentration en phospholipides utilisés pour l'étalemenl constituent des paramètres à prendre en compte des lors quäils conditionnent lit structuration de la monocouche lipidiqute (formation d'îlots lipidiques...) [6.7]. Finalement, ke prolocole expérimental utilisé pour réaliser nos monocouches est le suivant:

- la sous-phase est constiluéc d'eau pure milliQ el d’une solution tilmpon ( $\mathrm{NaCl} 0.1 \mathrm{M}$. Tris-HCl 2() $\mathrm{mM}$ ) pour fïxer le polentiel électrique de surlite el le pH.

- le solvant est constitué de chloroforme, d'hexane ou d'un mélange isovolume de cess solvants.

En prallique, deux cuves sont utilisées : une cuve de Linngmuir en Téflon (surface : $240 \mathrm{~cm}^{2}$ ) at une cuve de spectromítric destinée à la visualisation sous microscope (surfike: $1 \mathrm{~cm}^{2}$ ). Une altente de 30 minutes est respectée alprés dípô des phospholipides pour que le solvant s'évapore complètement el quie la monocouche s'homogénéise. Une cinćliquse d'évaporation trop rapide engendre des inhomogénéités dans la monocouche : iffin de la ralentir. un couvercle est placé sur la cuve. Les tïlms de phospholipides sont ensuite caractérisés mécaniquement à l'aide de courbes de compression obtenues sur cuse de Lilngmuir el optiquement via la microscopie par épillusorescence.

\section{- Courhes de compression sur cuve de Langmuir}

Les manipulattons sur cuve de Langmuir permettent de voir l'évolution de lat tension de surlate en fonction de lat compression 2-D de la monoxouche de phospholinides [8]. It est ainsi possible de relier la tension de surlace à la concentration interficciale en phospholipides. Lal cuve de Lingmuir est entièrement remplie par la sous-phase, lacpuelle est ensuite recouverte de sal monocouche. A lat surliace, une barrierre en Téllon mobile mouille le liquide el comprime lit monocouche en se déplaçant. La tension de surlace est simultinément mesuréc par une balance de Wilhelmy. Les solutions utilisées pour fiabricuter les monocouches sur la cuve de Langmuir sont généralement assez concentrées (2 $\mathrm{mg} / \mathrm{L}$ ) pour éviter d'alvoir une trop grande quitntité à déposer (typiquement 4 ou 5 lois $2 \mu \mathrm{L}$. Les phases gilzeuse. liquide el solide sont observées sur les courbes obtenues Vigure 9). Dans lit phatse gatzeuse, les phospholipides sont dispersés et interagissent peu entre eux. Danns ha phase liquide. laaire par molécule est un peu plus réduite et les phospholipides interagissen entre eux : la surfalce conserve une certaine thuidité. Lorsque lat compression de la monocouche est telie que la phalse solide est alteinte. les phospholipides son collés les uns aux autres. s'inclinent et leurs chaînes carbonées s'entrelicent. Au-delà, si lal compression persiste. la monocouche se déstructure (collapsel. Les isothermes de compression ont été réallisćes pour les DOPC, les BDPPE el le mélange BDPPE/DOPC permettant de fonctionnaliser l'interlace catt/air.

Pour des monocouche pures composées. soit de DOPC, soit de BDPPE, l'aire disponible par molécule varie entre $70 \AA^{2}$ et 2 10 $\AA^{2}$. Cependant, les deux types de phospholipides adoplent des comporlements différents durant lit compression puiscule les aires par molécule associces an collapse sont diffirentes : $70 \AA^{2}$ pour les DOPC. $120 \dot{A}^{2}$ pour les BDPPE. L'isotherıne de compression réaliscé sur monocouche mixte
BDPPE:DOPC 11 : tmole. figure 9) présente un Épitulement it une aire moléculatire - $I(x) \AA^{2}$. Si on eltectuc l'isotherme pour un mélinge ( 1 : I mole). l'épitulement. plus précoce el plus prononeć. survient à une aire par molécule - $120 \AA^{2}$ yui coïncide arec le collapse des BDPPE : il est donc possible que les phospholipides ne sorent pass miscibles.

\section{- Visualisation par fond moir et épifluorescence.}

Ces isothermes de compression ne donniant accies yu itux propriétés macroscopicues moyennées de lil monocouche, il est intéressint de mener une caractérisation plus microscopiyue par fond noir ou épitluorescence. La visualisation par fond noir permel de suivre l'élalement el l'évaporation du solvant. Lorsqu il est déposé sur une surfice acpueuse, le chloroforme a tendance à réticuler at la surlace de l'eau alors que l'hexane s'étale sur toute las surlace et forme un tïlm minee. Celte différence de comportement vient de lit solubilite du solvant dans leasu qui conditionne lat lension de surfice. Par exemple le chloroforme se dissoul suflisimmen dans l'eill pour salurer la surfice eilu/air é qui modilie l'équilibre des tensions superticielles et permet d'avoir un angle de contact non nul entre l'ealu et le chlorolorone (fommation d'une lentille en surfice). Ce phénomene n'est pas ohservé avec l'hexane dont la solubilité danns l'eau est hien plus laible. Par ailleurs. on observe lors de l'évaporation. un hratssage alu nivealu de l'interlace du a leffel Marangoni chimique ggradient en concentration interliaciale de phospholipides) el thermique (évapos)ration du solvant).

Une curatciérisation de la monexouche par épifluorescence est également réaliscéc. Pour celas. des phospholipides lluorescents (NBD-PE. Avanti Polar lipid) sont mélangés à des phospholipides classiugues (DOPC) el déposés sur un timpon (0.1 $\mathrm{M} \mathrm{NaCl} .20 \mathrm{mM}$ Tris $\mathrm{HCl}$ ). Lat visualisation de la surfiace révèle lit présence d'ìlots tluorescents très denses. compatibles avec la non-miscihilite suspectéc a l'issue des isothermes de compression. Les couches de phospholipides ainsi fithricucess se révèlent inhomogènes et la présence d'îlots constitue un réel probleme pour mesurer la cinćliçue d'adsorption de toute molécule cible. Plusieurs études porlint sur des phospholipides ont été réalisées en épiłluorescence dans la littérature [6.7. 9]. Toules montrent que lit monocouche obtente est effectivement composece de domaines plus ou moins condensés. Le comportement de la monocouche est complexe puisque selon son étal de compression et selon la nature des phospholipides. lit taille ou ke nombre de ces domaines changent.

\section{- III.3 Montage optique appliqué à l'adsorption de la streptavidine : expérience préliminaire}

Avec une seringue Hamilton, un volume de $3 \mu \mathrm{L}$ d une solution mixte de phospholipides (lipides ligands BDPPE lipides intercialants DOPC. $(1:-1)$ mol. 23() $\mu \mathrm{M})$ est déposé sur une sous-phase ayueuse constiluce de $0.8 \mathrm{ml}$. de solution timpon ( $\mathrm{NaCl}$ ().IM. Tris- $\mathrm{HCl} 2(1) \mathrm{m}$ ). Après 30 minutes d'altente, $2(k) \mu l$. de solution de streptavidine fluorescente (foumisseur: Molecular Probes) à $40 \mu \mathrm{M}$ sont in jectis dans la sous-phase. Lal courhe de cinélicue obtenue neest pass satisl' airsinte : l'émergence aléatoire d'agg gloméralts condensés de phospholipides thorescents dians le champ de la calméra perturbe la mesure (situration du capleur CCD, traitement d'image très délicall). Ce phénomène est attribué à la mobilité de l’interlace. La fonctionnalisattion n’élint pass homogène. le signal cilpté par la cilméra latérale est instiltionnaire el ne rend pats compte de la cinéticue d"adsorption. 


\section{IV a PHENOMENES DE DIFFUSION/ ADSORPTION DANS UNE MOUSSE, 2-D}

Paralkelement aux développerments expérimentaux. une approcte de modelisation pour une mousse 2-D a clé initice avec trois hypotheses fonditmentiales préalablement émises :

H1. les interfikes liquide/gazz conslitulives de la mousse sont dotées de sites récepteurs fontionnalisés pour adsorber des molécules cibles : ces sites récepteurs son1 uniformément distritues le long des intertates.

H2. Les phenomenes hydrodynamiques lels que i) le drainage des films interstiticls. ii) le pompage lié à l'eflet Marangoni chissiugue atux intertikes. iii) lat comvection des molécules cihles dans les tïlms ou les interfatess sont négligés.

H3. La diftusion inlerfaciale est égilement négligée.

La première étape de la modélisition consiste à calculer l'évolution de lat concentration en sibles dams les films interstiticls. Celte concentration dépend de la diffusion moleculate au sein de ces lilms et des phénomenes d'atusorption/ désorption aux interfaces functionnalisées. Dans notre cats. le principe de la fonctionnalisation des intertices est tel que la desorption est négligeable devant l'adsorption (liaison ligand-récepteur très énergétique). Par consécpuent. un saltend à ee que. progressivement. la concentration en cihles diminue dans les fïlms interstitiels jusqu à une valeur asymptotique preche de gero si lous les siles recepteurs ne sont pias silturés à lia lín du calcul $\left(\Gamma<\Gamma_{\infty}\right)$.

Les hypothèses $\mathrm{H} 2$ el $\mathrm{H}_{3} 3$ impliquent que la dilfusion (volumique) dans les tilms inlerstilicls est le seul mécinisme de transport moleculaire considéré. $\frac{\partial C}{\partial t}=D \Delta C$. avec $C\left(\right.$ moles $\left./ \mathrm{m}^{3}\right)$. lat concentration en molecules cibles et b) $\left(\mathrm{m}^{2} / \mathrm{s}\right)$, le coelficient de diffusion.

La loi constitutive de Liangenuir rend comple de ladsorption aux interfaces tonctionnalisies.

$\frac{d \Gamma}{d h}=k_{w+1} C_{s}\left(\Gamma_{\infty}-\Gamma\right)-k_{n f f} \Gamma$. avec $\Gamma_{\infty}$ (moles $\left./ m^{2}\right)$, la concentration maximale en molécules cibles adsorbées. suppposéc unilorme (HI), $\Gamma$ (moles $\left./ \mathrm{m}^{2}\right)$, la concentration de surtiace en sites occupés. $k_{m n}\left(\mathrm{~m}^{3} / \mathrm{m}\right.$ ole/s), le cueflicient diadsorption. $k_{\text {afff }}(1 / \mathrm{s})$. le coefficien de désorption. $C_{s}$ (mole/m $\left.)^{3}\right)$. la concentration volumique en cibles atu voisinage de l'interfiace. Les deux ćquations précédentes sont couplees via la lai de Fick. $\frac{d \Gamma}{d t}=-\left.D \nabla C\right|_{s} \cdot \mathbf{n}$. avec $\nabla C l, \mathbf{n}$. he llux solutal nomial à l'interlace. Le système est résolu à l'dide du logiciel alux éléments-linis PEMLAB ${ }^{\text {TN1 }}$ |10). Les valeurs catractéristiques utilisées dans les calculs. $D=10^{-10} \mathrm{~m}^{-1 / \mathrm{s}}, \quad k_{u, n}=1(x) \mathrm{m}^{3} / \mathrm{mole} / \mathrm{s}, \quad k_{1, f f}=10^{-5} \mathrm{I} / \mathrm{s}$. $\Gamma_{\infty}=10^{-x}$ mole $/ \mathrm{m}^{2}$, ont été obtenues à partir dexpériences réalisées dans la méthoxle classique de cilplure sur suppon solide. On liail dence l'hypothèse que l'on peut functionnaliser les interfaces impliqués dans la mousse de fiçon similaire a une interlace solide/liquide.

Du point de vue géométriguke le modèle proposé sapplique ì un réseau de bulles circulaires el fïxes. typiquement représentatif des mousses 2-D eau/air que nous atvons ubtenu expérimentialement a l'aide de la microscopie par épithuorescence vigure (0). Ces mousses sont slabilisées par ajout de

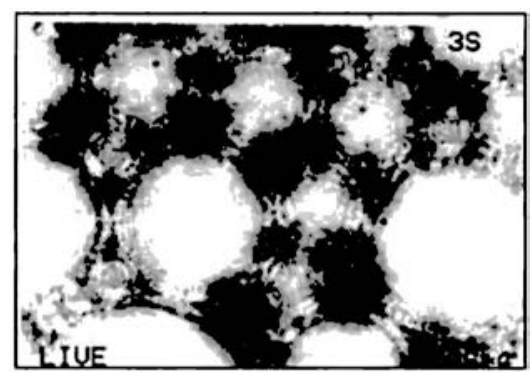

Figure 110. Observation par épifluorescence d'une mousse 2-I) eau/air.

La taille moyenne des bulles est d'environ $500 \mu \mathrm{m}$.

lensibatitl (Triton) et coincées entre deux placpues de verre. Les particules Transfluo permeltent de visualiser les films interstitiels el de valider l'hypothèse H2. En s"inspirant de la figure /0. on définit pour le calcul autant de régions que de bulles et une seule region pour tous les films interstiticls (domaine connexe). Puis le domaine de calcul est maillé avec des ádéments triangulaires de Delauncy. La figure // represente les isovaleurs en niveaux de gris de la concentration en cibles it 3 instants $t=1.10$. 100 secondes. Les bulles périphériques permetlent de montrer que l'épaisseur caracléristique de la couche limite diffusionnelle est commensurable avec les films minces insterstitiels. 11 n'est donc pas étonnant de constiter que l'adsorption est limitée dans les films les plus minces: lit composinte normale du gra-

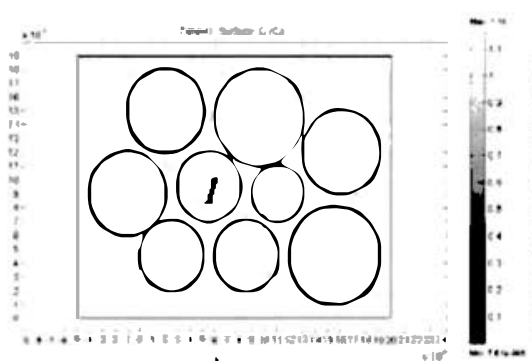

a)

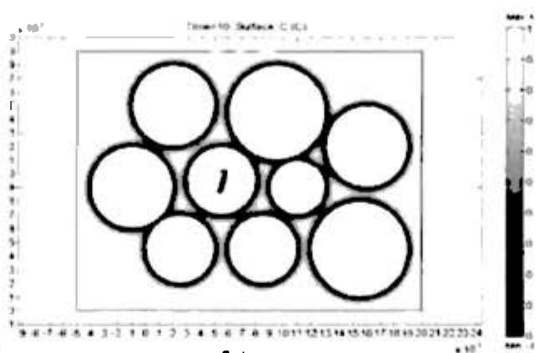

b)

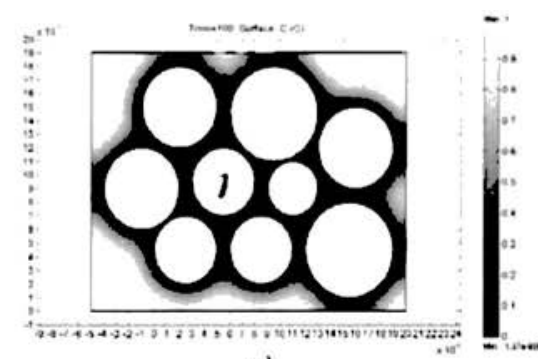

c)

Figure 11. Concentration volunique en molécules cibles dans les films minces, a) $t=\mathbf{t} \mathrm{s}$ : la diffusion $n$ 'est pas encore visible, b) $\mathbf{t}=10 \mathrm{~s}$ : la diffusion/adsorption commence à affecter unc zone circulaire autour des bulles, c) $t=100 \mathrm{~s}$ : la capture des molécules cibles est pratiquement tcrninée dans les films interstitiels entre les bulles. 


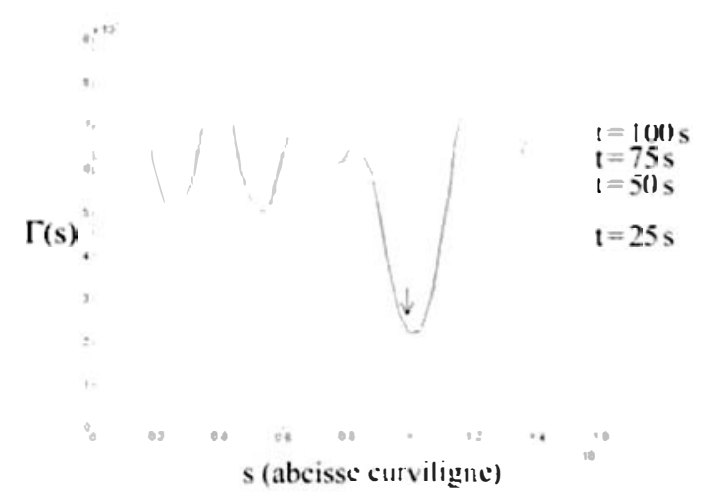

Figure 12. Profils de concentration $\Gamma(s)$ à la surface de la bulle $\mathrm{n}^{\circ} 1$ ( voir figure 11 ) aux temps $t=25,50,75$ et $100 \mathrm{~s}$. les petites fluctuations observées sur $\Gamma(s, t=25 \mathrm{~s})$ sont la signature d’un léger bruit numérique (effet des mailles). la flèche indique la plus faible valeur de $\Gamma$ qui coïncide avec la région la plus amincic d'un film interstiticl : la fin du régime transitoire y est rapidement atteinte, en moins de 25 secondes. les profils à $t=75 \mathrm{~s}$ et $100 \mathrm{~s}$ tendent à se superposer, ce qui montre que l'équilibre global est atteint au-delà de $100 \mathrm{~s}$.

dient solutal en molécules cibles s'y trouve plus laible. Ce phénomène peut être illustré quantitativement avec lat figure 12. A un instant t donne, on trace la distribution do concentration interfaciale $\Gamma(s)$ en molecules cibles adsorbies le long de l'interlace doune bulle-test (bulle $n^{\circ} 1$ sur la figure 1/1: celte opération est réiléréc à d'atulres instants durant le calcul. On observe une succession de bosses et de creux correspondants aux régions les plus larges ou les plus minces du lïm interstitiel enveloppant la bulle-lest. On observe yue les courbes se resserrent dans les creux conf irmant que léquilibre est rapidement alteint dans les régions les plus minces des films interstiticls tandis que les concentrations interfaciales y sont les plus faibles. En accord avec lat litlérature sur les mousses. la concentration interlaciale est trouvere maximale aux extrémilés des films instertiticls (ménisques de Plateatu).

\section{$V \square$ CONCLUSION ET PERSPECTIVES}

Le montage optique présenté dans cet article a éte entièrement caraclérisé (propriétés de l'éclairage, du dispositif de capture, trailement d'image) : il perme de mesurer avec une precision sulfisante la cincilique d'adsorption de particules ou molécules thuorescentes solubles ou amphiphiles à l'échelle microtluidique d"une interlace liquide/gaz.

Dans le cas ous une interface eau/air est fonctionnaliséc par des phospholipides. des difiticultés ont été mises en évidence quant a l'obtention d'une monocouche homogène. La lluidité de l'interface fonctionnalisce autorise la mobilite 2-D d'ilots denses en phospholipides lluorescents : ces ilots sont capables de provoquer une augmentation soudaine du signal de fluorescence émise lorsqu ils croisent le faiscean lumineux d'excitation. Le signal reçu par la cauméra latérale est donc instationnaire el ne peut rendre comple de la cincitique d'adsorption.

La cuve de spectroscopie utilisce jusqu alors dans nos experiences devra donc être transformée en une cuve de Langmuir permettant la compression progressive de lat monocouche de phospholipides et laapplication simultance de la méthode de caracterisation opticute proposse dans cet article. Il devrait donce ètre possible de connaitre les conditions de température el de pression de la monoxouctie qui rendent l'interface linnetionnaliséc homogène. Dans ce cats seulement. la mesure de ladsorption de la streptasidine a une interface catu/air fonctionnalisece par des phospholipides est envisageable.

En accord avec la litterature sur les mousses, ha mexlélisation du transport par diffusion/adsorption alu sein des tilms minces interstiticls d'une mousse ciutu/air 2-D at mis en evidence une densilication des molécules cibles adsorbés au nivealu des régions de Platcialu. Outre le lait que leflet Marangoni chimicue nesi pas inclus dans la modelisation tphénomene pourtant susceptible de sopposer à la densilicaltion lociale de cibles adsorbeies), le modele mis en reuvre se base sur des hypothèses simplificatrices portant notamment sur l'absence de convection el sur une fonctionnalisation hourogene des interlaces. Néanmoins, on obtient un orslee de grandeur du transitoire nécessaire à la diffusion/addsorption complète de loordre de $I(x)$ secondes pour le eals d'une fonctionnalisation d’interlaces liquide/gar équivalente à celle d'une puce solide.

Parmi les compléments utiles ì celte modélisattion. il parait opportun denvisager à terme le couplage avec la microtluidicyue dans les lilms interstiticls. couplage yui appatrait à traters le bilan des contraintes aux interfaces el la rhéologie interlaciale.

\section{BIBLIOGRAPHIE}

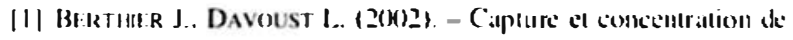
inolécules par émulsion dans un inicro- réacteur. Application a

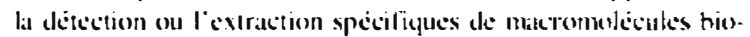
logiques ou particules colloridiales. Brevel CLACCNRS $10^{\circ}$ DD2319 citendu.

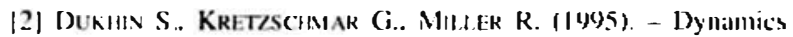
of Aclsorption al Liquid Interfiaces. Fisevier.

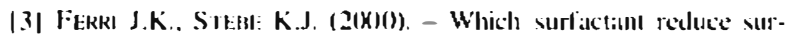
late tension faster. Adv. in Colloid \& lut. Sci.. 85. pp. 61-97.

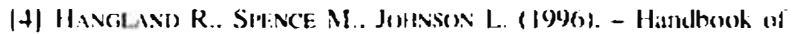
thoorescent Prohes and Resciarch Chemicals. fieme edition.

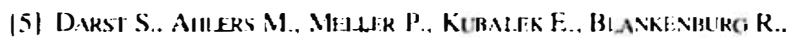

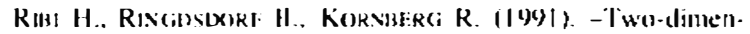
sional erystals of streptavidin on hiolinylated lipid layers and lheir interaclions with hiolinylated macromolecules. Biophys. J. 59. pp. $3 \times 7.390$.

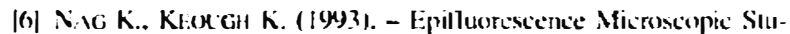
dies of Monolayers Containing Mixtures of Dioleoyl- and Depalmitoylphosphatidylcholunes, Biophss. J. 65. pp. I(1)14-18126.

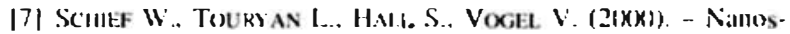
cale Topographic lustahilities of a plosspholipid .Monolayger. J Phys. Chem., 114. pp. 7.38x.7393.

|x| Niwa. L.angmuir Bloxlgetl troughs, Operating Manual

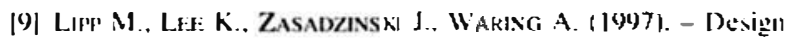
and pertiormance of an integrated thuorescence. polarized fluserescence. and Brewster ingle nicioscope/Lattgmuir trough assembly for the study of lumg momolityers. Rev. Sci. [nst.. 68, pp. $257 \dot{4}-2582$

[II) F FEMLAB. (2(X)2). - Multiphysics in MATt,AB. Version 2.2.. http://www. femlaboum 